\title{
Twenty Years of Breast Reduction Surgery at a Veterans Affairs Medical Center
}

\author{
Loretta Coady-Fariborzian, MD; and Christy Anstead, ARNP-BC
}

\begin{abstract}
Background: Breast reduction surgery has a high patient satisfaction rate for the treatment of symptomatic macromastia. However, complications from the surgery can significantly disrupt a woman's life due to time in the hospital, clinic appointments, wound care, time off work, and poor aesthetic outcome. Beginning July 2007, the Malcom Randall Veterans Affairs Medical Center (MRVAMC) Plastic Surgery Service in Gainesville, Florida, started using a preoperative screening protocol to help patients achieve a healthier and more favorable risk profile.

Methods: A retrospective chart review was conducted on all breast reduction surgeries performed at the MRVAMC from July 1,2000 to June 30, 2020. Medical records were queried for all primary breast reduction surgeries performed for symptomatic macromastia. Potentially modifiable or predictable risk factors for wound complications were recorded: nicotine status, body mass index (BMI), diabetes mellitus (DM) status, skin incision pattern, and pedicle location. Records were reviewed for 3 months after surgery for local wound complications that
\end{abstract}

included: hematoma, infection, wound breakdown, skin and nipple necrosis. Major complications required an unplanned hospital admission or operation.

Results: Over the 20-year period, 115 bilateral breast reduction surgeries were performed. There were 48 wound complications $(41.7 \%)$ and 8 major complications $(7 \%)$. Most complications were identified in the first 7 years of the study. BMI $>32(P=.03)$ and active nicotine use $(P=.004)$ were found to be statistically significant risk factors for wound complications. DM status $(P=$ $.22)$, skin incision pattern $(P=.25)$, and pedicle location $(P=.13)$, were not predictors of wound complications.

Conclusions: Breast reduction surgery has a high wound complication rate, which can be predicted and improved upon so that patients can receive their indicated surgery with minimal inconvenience and downtime. This review confirms that preoperative weight loss and nicotine cessation were the appropriate focus of the MRVAMC Plastic Surgery service's efforts to achieve a safer surgical experience.
W omen make up an estimated 10\% of the veteran population. ${ }^{1}$ The US Department of Veterans Affairs (VA) projected that there would be an increase of 18,000 female veterans per year for 10 years based on 2015 data. The number of women veterans enrolled in the VA health care increased from 397,024 to 729,989 (83.9\%) between 2005 and $2015 .{ }^{2}$ This rise in the number of enrolled women veterans also increased the demand for female-specific health care services, such as breast reduction surgery, a reconstructive procedure provided at the Malcom Randall VA Medical Center (MRVAMC) federal teaching hospital in Gainesville, Florida.

Patients who experience symptomatic macromastia will report a history of neck and shoulder pain, shoulder grooving from bra straps, inframammary intertrigo, difficulty finding clothes that fit, and discomfort participating in sports. For the treatment of symptomatic macromastia, patients report a high satisfaction rate after breast reduction surgery.-5 Unfortunately, the complications from the surgery can significantly disrupt a woman's life due to previously unplanned hospital admissions, clinic appointments, wound care, time off work, and poor aesthetic outcome. Fac- ulty awareness of a large number of complications for patients after breast reduction surgery prompted the MRVAMC Plastic Surgery Service to establish a stricter surgical screening protocol using body mass index (BMI) values and negative nicotine status to help patients be healthier and reduce the potential risk before offering surgery. A medical literature search did not find an existing study on veteran-specific breast reduction surgery.

\section{METHODS}

The University of Florida and North Florida/South Georgia Veterans Health System Institutional Review Board approved a retrospective chart review of all breast reduction surgeries performed at MRVAMC over a 20-year period (July 1, 2000-June 30, 2020). Electronic health records were queried for all primary bilateral breast reduction surgeries performed for symptomatic macromastia using Current Procedural Terminology code 19318. Potentially modifiable or predictable risk factors for wound complications were recorded: nicotine status, BMI, diabetes mellitus (DM) status, skin incision pattern, and pedicle location. Skin incision patterns were either vertical (periareolar plus a vertical scar from
Loretta Coady-Fariborzian is the Plastic Surgery Section Chief, and Christy Anstead is a Nurse Practitioner in the Plastic Surgery Service, both at Malcom Randall Veterans Affairs Medical Center in Gainesville, Florida. Loretta Coady-Fariborzian is Associate Professor of Plastic Surgery at the University of Florida. Correspondence: Loretta Coady-Fariborzian (Imcoady@aol.com)

Fed Pract. 2021;38(7). Published online July 12. doi:10.12788/fp.0150 
FIGURE 1 Wise Pattern SuperiorMedial Pedicle Breast Reduction
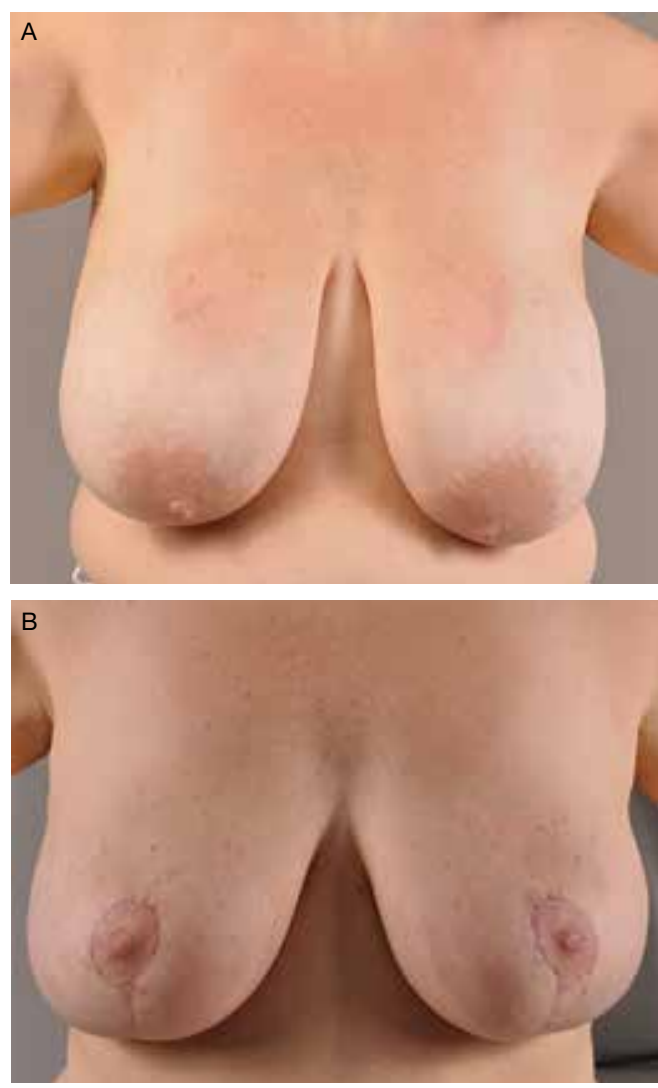

A, preoperation; B, 6-weeks postoperation, $1285 \mathrm{~g}$ total reduction.

the areola to the inframammary fold) or traditional Wise pattern (also known as anchor pattern: periareolar scar, vertical scar to inframammary fold, plus a horizontal scar along the inframammary fold) as seen in Figures 1 and 2. The pedicle is the source of blood supply to the nipple, which was documented as either from the inferior aspect or the superior or superior/medial aspect.

For this study, the blood supply from the superior and superior/medial was logged in the same category. Records were reviewed 3 months after surgery for documentation of local wound complications, such as hematoma, infection, wound breakdown, skin necrosis, and nipple necrosis. Major complications were defined as requiring an unplanned hospital admission or urgent return to the operating room. A $\chi^{2}$ test using a $P$ value of $<.05$ was used to determine statistical significance between the incidence of

\section{FIGURE 2 Wise Pattern Inferior Pedicle Breast Reduction}
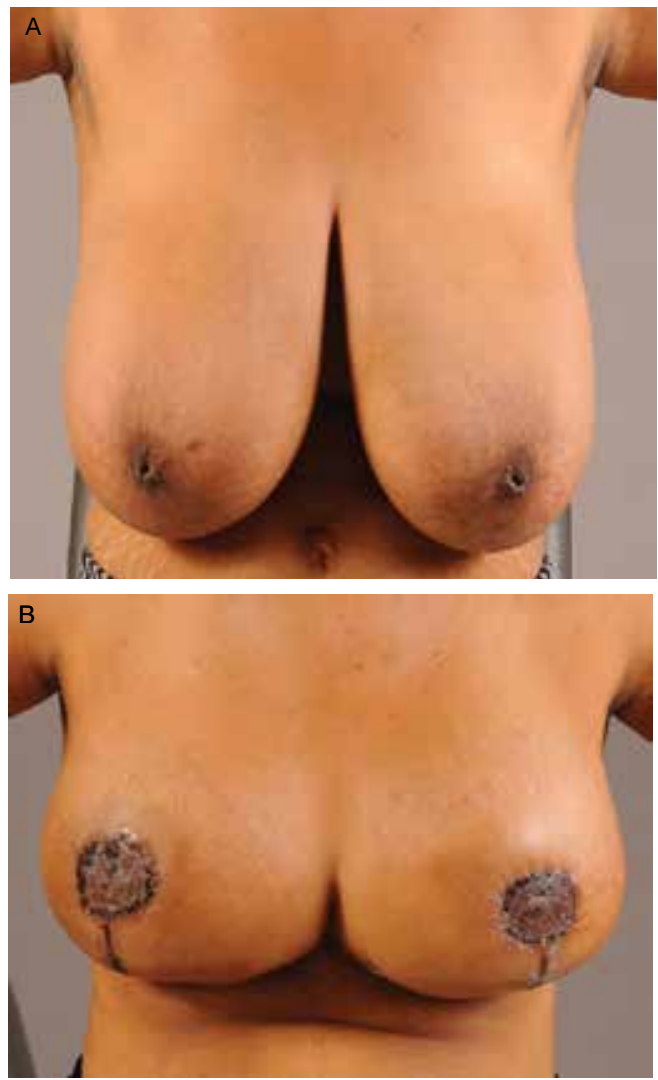

A, preoperation; B, 2-week postoperation, $1826 \mathrm{~g}$ total reduction.

wound complications and the individually identifiable variables.

\section{RESULTS}

One hundred fifteen bilateral breast reduction surgeries were performed at MRVAMC over a 20 -year period. Patient median age was 43 years. Median combined specimen weight was $1272 \mathrm{~g}$. Forty-eight (41.7\%) wound complications were documented, including 8 (7\%) major complications. Most complications were identified in the first 7 years of the study before the new protocol and consult template became active. The new template resulted in the local complication rate dropping from $62 \%$ (July 2000-June 2007) to 26\% (July 2007-June $2020)$. BMI $>32(P=.03)$ and active nicotine use $(P=.004)$ were found to be statistically significant independent risk factors for wound complications. Median BMI for all patients was 30 . DM status $(P=.22)$, 
skin incision pattern $(P=.25)$, and pedicle location $(P=.13)$ were not found to be predictors of wound complications (Table). There was no significant change in the incidence of major complications before and after the new protocols were enforced.

\section{DISCUSSION}

Breast reduction surgery is an elective reconstructive option to treat symptomatic macromastia. There are several accepted ways to do the reduction surgical procedure where the blood supply (pedicle) to the nipple can vary and the visible scars can be in a horizontal, vertical, or Wise pattern. Technique is usually based on surgeon training, comfort, and preference. There are several known complications specific to this operation that include asymmetry, changes in nipple sensation, unattractive scars, diminished ability to breastfeed, and wound complications. . $^{5-7}$ Wound complications include seroma, hematoma, dehiscence, infection, wound breakdown, skin necrosis, and nipple necrosis.

This study focused on wound complications with the objective of identifying and modifying risk factors. Two known risk factors documented in the literature, nicotine use and obesity, already had been addressed by our service, and results were known anecdotally but had not been previously verified. This study also looked at other potential risk factors, including the pedicle location, skin incision, and DM status.

Residents or fellows participated in all the surgeries. An outcome analysis from The American College of Surgeons National Surgical Quality Improvement Program database from 2005 to 2011 found that resident participation was associated with morbidity, including wound complications. ${ }^{8}$ This study was performed at a federal hospital with a complexity level la rating, which is designated based on the highest level of patient volume, risk, teaching, research, intensive care unit beds, and specialty services. ${ }^{9}$ The hospital is closely affiliated with a level 1 trauma center and teaching hospital; therefore, resident and fellow participation is not a modifiable risk factor.

This study did not find an increased risk of wound complications in patients with DM, which has been found to be an independent risk factor in a prior study. ${ }^{10} \mathrm{DM}$ status was
TABLE Risk Factors for Breast Reduction Wound Complications

\begin{tabular}{|c|c|c|c|}
\hline Variables & Patients, No. & Complication, No. & $P$ value \\
\hline Current nicotine use & & & $.004^{a}$ \\
\hline $\begin{array}{l}\text { Yes } \\
\text { No }\end{array}$ & $\begin{array}{c}14 \\
101\end{array}$ & $\begin{array}{l}11 \\
37\end{array}$ & \\
\hline Body mass index & & & $.03^{a}$ \\
\hline$>32$ & 27 & 16 & \\
\hline$\leq 32$ & 88 & 32 & \\
\hline Diabetes mellitus status & & & .22 \\
\hline Diagnosed & 3 & 2 & \\
\hline Not diagnosed & 112 & 46 & \\
\hline Scar pattern & & & .25 \\
\hline Wise & 69 & 32 & \\
\hline Vertical & 46 & 16 & \\
\hline Pedicle & & & .13 \\
\hline Inferior & 52 & 26 & \\
\hline Superior or superior/medial & 63 & 22 & \\
\hline
\end{tabular}

aStatistically significant.

indicated in only 3 histories, and they all had perioperative hemoglobin $A_{1 c}$ levels $<8 \%$. There is documentation of patients receiving perioperative antibiotics in 99 out of 116 of the surgical records; however, we did not include this in the analysis because the operative reports from the first year of the study were incomplete.

Smoking is a known risk factor for local wound complications in breast reduction surgery. ${ }^{10-15}$ The VA has a smoking cessation program through its mental health service that provides counseling and medication treatment options, including nicotine replacement, bupropion, and varenicline. We require patients to be at least 4 weeks nicotine free before surgery, which has been previously recommended in the literature. ${ }^{16}$

Existing studies that compare the traditional Wise pattern/inferior pedicle with vertical pattern/superior medial pedicle did not find an increased risk of wound complications. ${ }^{17-19}$ Our study separated the different incisions from the pedicle because the surgical technique among the different surgeons in the study varied, where sometimes the traditional Wise pattern was combined with the less traditional superior-medial pedicle. We did not find a statistical difference when comparing the incisions and pedicle location, which suggests that the incision type and source of blood supply to 
the nipple are not the determining factors for wound complications in the early postoperative period.

Obesity is a known risk factor for local wound complications. ${ }^{12,13,15,20-22}$ Studies have shown that patients who are obese benefit from breast reduction surgery; authors have argued against restricting surgery to these higher risk patients. ${ }^{4,23-25}$ Patients usually report decades of macromastia symptoms at consultation; so, we believe delaying the surgical procedure to get patients to a safer risk profile is in their best interest. We chose a cutoff BMI of 32 as a realistic value rather than 30, which is considered the definition of obesity. Patients at MRVAMC have access to MOVE!, a weight loss management program through primary care. We believe in being reasonable; so if a patient makes a significant improvement in her health but falls short of the required cutoff, we will still consider offering the surgical procedure after a full explanation of the surgical risks.

Wound complications, especially those that require admission or frequent appointments can seriously disrupt a patient's life, creating unnecessary hardships and expense in time lost from work, travel, and child care. MRVAMC has a catchment area the size of North Carolina; so many of our patients travel hours for their appointments. The added scars and deformity from wound dehiscence and debridement can lead to asymmetry, widened scars, and future revision operations. Multiple clinic appointments for wound care not only impact that individual patient, but also has the effect of limiting access for all patients in a health care environment with high patient volume and limited providers, operating room time, and clinic appointments. As a result, minimizing predictable wound complications benefits the entire system.

\section{Limitations and Strengths}

This retrospective review comprised multiple different surgeons, including faculty and trainees, who were involved in the consultation, surgery, and postoperative care of the patients over a 20-year period; therefore, consistency in documentation is lacking. In addition, we were limited to only the information available on the charts. For example, wound size and laterality were not consistently doc- umented. The MRVAMC complication rate was consistent with the current literature (range, 14-52\%). ${ }^{12,18,20,24}$

The major strength of the study is that the veterans tend to stay within the VA, which makes complications easier to identify and follow. Patients who do not present initially to their surgeon due to travel limitations will typically contact their primary care provider or present to their local VA urgent care or emergency department provider, who will route the patient back to the surgical specialty service through the electronic health record.

\section{CONCLUSIONS}

Breast reduction surgery has a high wound complication rate, which can be predicted and improved on so that patients can receive their indicated surgical procedure with minimal inconvenience and downtime. This review confirms that preoperative weight loss and nicotine cessation were the appropriate focus of the MRVAMC plastic surgery service's efforts to achieve a safer surgical experience. We will continue to enforce our protocol and encourage patients who are interested in breast reduction surgery and fall outside the requirements to work with their primary care provider on smoking cessation and weight loss through better nutrition and physical activity.

\section{Acknowledgment}

This manuscript is the result of work supported with resources and the use of facilities at the North Florida/South Georgia Veterans Health System in Gainesville, Florida.

\section{Disclosures}

The authors report no actual or potential conflict of interest with regard to this article.

\section{Disclaimer}

The opinions expressed herein are those of the authors and do not necessarily reflect those of Federal Practitioner, Frontline Medical Communications Inc., the U.S. Government, or any of its agencies.

\section{References}

1. US Department of Veterans Affairs. Statistics at a glance. Published February 2020. Accessed June 18, 2021. https://www.va.gov/vetdata/docs/Quickfacts/Homepage slideshow_4_6_20.PDF

2. US Department of Veterans Affairs, National Center for Veterans Analysis and Statistics. Women veterans report: the past, present, and future of women veterans. Published February 2017. Accessed June 18, 2020. https://www.va.gov/vetdata /docs/specialreports/women_veterans_2015_final.pdf

3. Crittenden TA, Watson DI, Ratcliffe J, Griffin PA, Dean NR. Outcomes of breast reduction surgery using the breastq: a prospective study and comparison with norma- 
tive data. Plast Reconstr Surg. 2019;144(5):1034-1044. doi:10.1097/PRS.0000000000006114

4. Thoma A, Sprague S, Veltri K, Duku E, Furlong W. A prospective study of patients undergoing breast reduction surgery: health-related quality of life and clinical outcomes. Plast Reconstr Surg. 2007;120(1):13-26. doi:10.1097/01.prs.0000263370.94191.90

5. Nuzzi LC, Firriolo JM, Pike CM, DiVasta AD, Labow BI. Complications and quality of life following reduction mammaplasty in adolescents and young women.Plast Reconstr Surg. 2019;144(3):572-581. doi:10.1097/PRS.0000000000005907

6. Hall-Findlay EJ, Shestak KC. Breast reduction. Plast Reconstr Surg. 2015;136(4):531e-544e. doi:10.1097/PRS.0000000000001622

7. Kraut RY, Brown E, Korownyk C, et al. The impact of breast reduction surgery on breastfeeding: systematic review of observational studies. PLoS One. 2017;12(10):e0 186591. doi:10.1371/journal.pone.0186591

8. Fischer JP, Wes AM, Kovach SJ. The impact of surgical resident participation in breast reduction surgery-outcome analysis from the 2005-2011 ACS-NSQIP datasets. J Plast Surg Hand Surg. 2014;48(5):315-321. doi:10.3109/2000656X.2014.882345

9. Site Facility Name and Complexity Summary of VHA Facility. Accessed June 18, 2021. https://www.vendorpor tal.ecms.va.gov/FBODocumentServer/DocumentServer .aspx?Documentld=2793591\&FileName=VA118-16 -R-1059-A00002002.docx

10. Lewin R, Göransson M, Elander A, Thorarinsson A, Lundberg J, Lidén M. Risk factors for complications after breast reduction surgery. $J$ Plast Surg Hand Surg. 2014;48(1):10-14. doi:10.3109/2000656X.2013.791625

11. Cunningham BL, Gear AJ, Kerrigan CL, Collins ED. Analysis of breast reduction complications derived from the BRAVO study. Plast Reconstr Surg. 2005;115(6):15971604. doi:10.1097/01.prs.0000160695.33457.db

12. Karamanos E, Wei B, Siddiqui A, Rubinfeld I. Tobacco use and body mass index as predictors of outcomes in patients undergoing breast reduction mammoplasty. Ann Plast Surg. 2015;75(4):383-387. doi:10.1097/SAP.0000000000000192

13. Manahan MA, Buretta KJ, Chang D, Mithani SK, Mallalieu $\mathrm{J}$, Shermak MA. An outcomes analysis of 2142 breast reduction procedures. Ann Plast Surg. 2015;74(3):289292. doi:10.1097/SAP.0b013e31829d2261

14. Hillam JS, Borsting EA, Chim JH, Thaller SR. Smoking as a risk factor for breast reduction: an analysis of 13,503 cases. J Plast Reconstr Aesthet Surg. 2017;70(6):734740. doi:10.1016/j.bjps.2016.12.012

15. Zhang $M X$, Chen $C Y$, Fang $Q Q$, et al. Risk fac- tors for complications after reduction mammoplasty: a meta-analysis. PLoS One. 2016;11(12):e0167746. doi:10.1371/journal.pone.0167746

16. Sørensen LT. Wound healing and infection in surgery: the pathophysiological impact of smoking, smoking cessation, and nicotine replacement therapy: a systematic review. Ann Surg. 2012;255(6):1069-1079. doi:10.1097/SLA.0b013e31824f632d

17. Antony AK, Yegiyants SS, Danielson KK, et al. A matched cohort study of superomedial pedicle vertical scar breast reduction (100 breasts) and traditional inferior pedicle Wise-pattern reduction (100 breasts): an outcomes study over 3 years. Plast Reconstr Surg. 2013;132(5):1068-1076. doi:10.1097/PRS.0b013e3182a48b2d

18. Hunter-Smith DJ, Smoll NR, Marne B, Maung H, Findlay MW. Comparing breast-reduction techniques: time-to-event analysis and recommendations. Aesthetic Plast Surg. 2012;36(3):600-606. doi:10.1007/s00266-011-9860-3

19. Ogunleye AA, Leroux O, Morrison N, Preminger AB. Complications after reduction mammaplasty: a comparison of wise pattern/inferior pedicle and vertical scar/superomedial pedicle. Ann Plast Surg. 2017;79(1):13-16. doi:10.1097/SAP.0000000000001059

20. Bauermeister AJ, Gill K, Zuriarrain A, Earle SA, Newman MI. Reduction mammaplasty with superomedial pedicle technique: a literature review and retrospective analysis of 938 consecutive breast reductions. J Plast Reconstr Aesthet Surg. 2019;72(3):410-418. doi:10.1016/j.bjps.2018.12.004

21. Nelson JA, Fischer JP, Chung CU, et al. Obesity and early complications following reduction mammaplasty: an analysis of 4545 patients from the 2005-2011 NSQIP datasets. J Plast Surg Hand Surg. 2014;48(5):334-339. doi:10.3109/2000656X.2014.886582

22. Kreithen J, Caffee H, Rosenberg J, et al. A comparison of the LeJour and Wise pattern methods of breast reduction. Ann Plast Surg. 2005;54(3):236-241. doi:10.3109/2000656X.2014.886582

23. Güemes A, Pérez E, Sousa R, et al. Quality of life and alleviation of symptoms after breast reduction for macromastia in obese patients: is surgery worth it? Aesthetic Plast Surg. 2016;40(1):62-70. doi:10.1007/s00266-015-0601-x

24. Setälä L, Papp A, Joukainen S, et al. Obesity and complications in breast reduction surgery: are restrictions justified? J Plast Reconstr Aesthet Surg. 2009;62(2):195-199. doi:10.1016/j.bjps.2007.10.043

25. Shah R, Al-Ajam Y, Stott D, Kang N. Obesity in mammaplasty: a study of complications following breast reduction. J Plast Reconstr Aesthet Surg. 2011;64(4):508-514. doi:10.1016/.j.bjps.2007.10.043 